Original Article

\title{
Ascorbic Acid Levels in Systemically Healthy Patients with and Without Periodontitis
}

\author{
Amitha Ramesh ${ }^{1}$, Agumbe Priyanka Prakash ${ }^{2}$, Arya Ashok $^{3}$, Biju Thomas $^{4}$ \\ ${ }^{1}$ Professor, ${ }^{2}$ Post Graduate, ${ }^{3}$ Intern, ${ }^{4}$ Head of the Department, Department of Periodontics, A B Shetty M emorial Institue of \\ Dental Sciences, M angalore \\ *Corresponding Author : Agumbe Priyanka Prakash, Post Graduate, Department of Periodontics, A.B. Shetty Memorial Institue of \\ Dental Sciences, M angalore, M obile : +91 9449752055, E-mail : agumbe.priyanka@yahoo.co.in
}

Received : : 30.01.2016

Review Completed : 05.05.2017

Accepted : :06.05.2017

Keywords : Ascorbic acid, chronic periodontitis, Antioxidant

\begin{tabular}{|c|}
\hline Access this article online \\
\hline Quick Response Code \\
\hline
\end{tabular}

\begin{abstract}
:
Introduction : Periodontitis is a multifactorial disease initiated by plaque. This slowly progressing disease affects the supporting structures of the teeth leading to attachment and bone loss. Nutrition plays an important role in the inflammation and this could be related to effect of nutrition on periodontal disease prevalence. Vitamin C also known as ascorbic acid is abundantly found in fruits and vegetables play a role in the periodontal disease. It can scavenge free oxygen radicals and protect lipid from peroxidation and is a potent antioxidant.
\end{abstract}

Materials and methods : A total of 50 systemically healthy subjects were selected and divided into control group A (25 subjects without chronic periodontitis) and test group B ( 25 subjects with chronic periodontitis) and blood samples were collected to assess ascorbic acid levels. The data obtained were subjected to statistical analysis.

Results : showed statistical significance ( $p$ value 0.004 ) in the ascorbic acid levels between the two groups.

Conclusion : The plasma ascorbic acid level was significantly reduced in systemically healthy individuals with chronic periodontitis when compared to systemically healthy without periodontitis which could be attributed to the protective nature of this vitamin in chronic disease like periodontitis.

\section{Introduction}

Periodontitis is a multifactorial disease initiated by plaque. This slowly progressing disease affects the supporting structures of the teeth leading to attachment and bone loss. Periodontal inflammation results in gingival bleeding, pocket formation, destruction of alveolar bone, and eventually loss of teeth. ${ }^{1}$

Nutrition plays an important role in the inflammation and this could be related to effect of nutrition on periodontal disease prevalence. Vitamin C also known as ascorbic acid is abundantly found in fruits and vegetables play a role in the periodontal disease. ${ }^{2}$ Vitamin $\mathrm{C}$ is a powerful water soluble antioxidant. It can scavenge free oxygen radicals and protect lipid from peroxidation. It is also shown to be useful in regeneration of a-tocopherol. ${ }^{3}$

Even though low vitamin $C$ intake does not cause periodontitis, an inverse association exists between plasma/serum vitamin C concentration and periodontitis ${ }^{4}$. Worsening of periodontal disease with development of scurvy has been reported. But epidemiological surveys have failed to establish a causal relationship between severity of periodontal disease and vitamin Clevels. ${ }^{5,6}$

Free radicals play an important role in the pathogenesis of periodontal disease. Vitamin $\mathrm{C}$ can scavenge free radicals mainly hydroxyl radicals. Vitamin C can prevent activation of neutrophils and gingival crevicular fluid derived collagenase by oxidative agent.

Collagen plays an important role in wound healing by collagen synthesis. This function of vitamin $\mathrm{C}$ helps in maintaining periodontal tissues, formation of bone matrix and also helps maintaining the blood vessel wall integrity. ${ }^{8}$ Ascorbic acid play a role in decreasing the gingival epithelial 
permeability and thus prevents the penetration of toxic substances into the periodontal tissues.

Hence the present study was done to assess and compare the serum ascorbic acid in systemically healthy subjects with and without periodontitis.

\section{Materials and Methods}

The present study included 50 individuals visiting the department of Periodontics, A.B Shetty M emorial Institute of Dental Sciences, M angalore, India.

The study was approved by the ethical committee of the institution.Written informed consent was obtained from all the patients after a detailed explanation of the procedures and objectives of the study. Data regarding the personal history, medical, dental, habit was recorded.

Clinical evaluation to determine the periodontal status of patients included assessment of plaque index by Silness and Loe (PI), gingival index (GI land mean pocket probing depth(PPD).Periodontal examination was done by a trained examiner and one calibrated examiner obtained all the measurements to reduce intra-examiner variability.

Patients were divided in to two groups:

- Group A (Control): 25 systemically healthy Subjects without chronic periodontitis

Group B (Test group): 25 systemically healthy Subjects with chronic periodontitis.

Selection criteria for the test and control groups were as follows.

\section{Inclusion Criteria}

- Patients between 35-55 yrs of age

- Patients with minimum complement of 20 teeth

- Patients with gingival index score(Loe and Silness, 1963) of $<1$ for Group I

- Patients with Gingival Index score between 1-2(Loe and Silness, 1967) and probing depth of $\geq 4 \mathrm{~mm}$ in more than $30 \%$ of the sites for Group II.

\section{Exclusion Criteria}

- History of any antibiotic or anti inflammatory therapy in the past 6 months
- History of any systemic disease

- Pregnant or lactating women

- Subjects with any prior history of periodontal therapy in the last 6 months

- Smokersand tobacco use.

Collection of samples: Blood samples were collected from the participants into $5 \mathrm{ml}$ vacuum tubes. The collected samples were centrifuged with a low speed centrifuge at $4000 \mathrm{rpm}$ for 5 minutes, to separate plasma from blood cells. The supernatants were immediately stored in $4^{\circ}$ Celsius to be used later to determine the plasma vitamin $\mathrm{C}$ levels by spectro photometry.

\section{Statistical analysis}

The data was analysed using statistical software package version. Chi square tests, Students t-tests and Fishers exact tests were used to assess the statistical significance of the study. Clinical parameters of the test group were calculated in mean scores. Student's t-test was used to compare the differences between mean values.

P values of $\varangle 0.05$ were accepted as statistically significant.

\section{Results}

The sample size of this study was 50 , which were divided into two equal groups, group $A$, being the control (n-25) and group B- systemically healthy subjects with chronic periodontitis $(n-25)$. The ascorbic acid levels were calculated for all the subjects. The ascorbic acid level of the test group was compared with each clinical parameter. (Table 2)

The comparison of ascorbic acid levels between group A and group B showed statistical significance ( $p$ value 0.004 ) as shown in table 1 and figure 1.The comparison of levels of ascorbic acid of group B with GI was found statistically significant ( $p$ value $=0.035$ ) whereas between PI ( $p$ value $=0.6$ ) and PPD ( $p$ value=1) were not statistically significant (table 2). 
Table 1 : Vitamin C levels in Group A and B

\begin{tabular}{|c|c|c|c|c|}
\hline GROUPS & MEAN & $\begin{array}{r}\text { STANDARD } \\
\text { DEVIATION }\end{array}$ & t value & p value \\
\hline A & 0.672 & 0.232 & 3.009 & $0.004^{*}$ \\
\hline B & 0.500 & 0.16 & & \\
\hline
\end{tabular}

Statistically significant with $p \varangle 0.05$

Table 2 : Independent sample test showing comparison of Vitamin C in Test group (Group B) with PI (Plaque Index), GI (Gingival Index) and PPD (Probing Pocket Depth)

\begin{tabular}{|l|c|c|c|}
\hline & PI & GI & PPD \\
\hline Vitamin C & 0.667 & $0.035^{*}$ & 1.0 \\
\hline
\end{tabular}

Statistically significant $p \varangle 0.05$

Figure 1: Vitamin C levels (mg/dl) in Group A and Group B

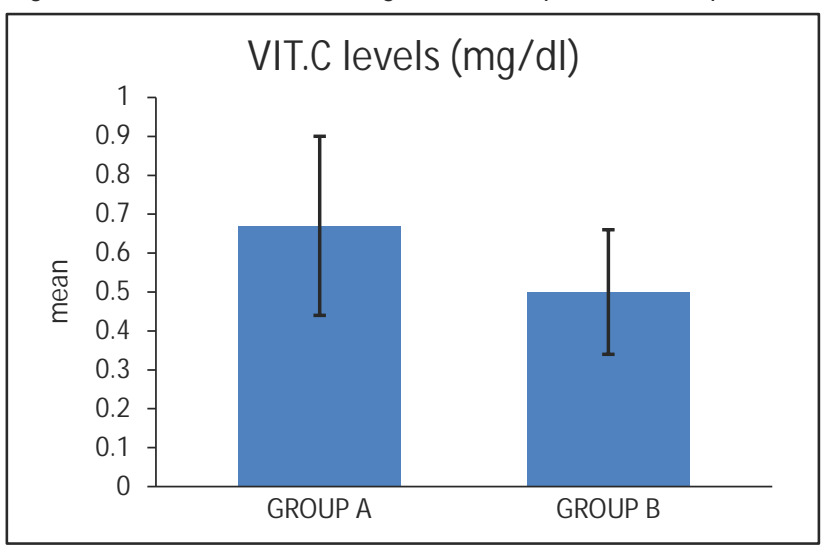

M ean \pm Standard deviation of Vitamin C in Group A Group B were $0.672 \pm 0.23$ and $0.5 \pm 0.16$ respectively.

\section{Discussion}

Free radicals induce lipid peroxidation which leads to various pathological changes in the periodontium leading to periodontitis. Reactive oxygen species (ROS) are continuously produced by our body during metabolism. ${ }^{9}$ if produced in excess can lead to harmful damage to the periodontium. Our body produces an array of antioxidants to fight against this harmful free radicals. ${ }^{10}$ Vitamin $\mathrm{C}$ is a non enzymatic antioxidant. Vitamin $\mathrm{C}$ is a powerful water soluble antioxidant. It can scavenge free oxygen radicals and protect lipid from peroxidation. It is also shown to be useful in regeneration of a-tocopherol. ${ }^{3}$

There exists a synergy between antioxidants. It is shown

\section{References}

1. Robert JG. Current view of risk factors for periodontal diseases. J Periodontaal October 1996; 67: 1041-9.

2. Woolfe SN, Hume WR, Kenny EB. Ascorbic acid and periodontal disease: a review of the literature. J West Soc Periodontal Abstr 1980; 29:44-56.

3. Padh H. Vitamin C: never insights into its biochemical that dietary supplementation of vitamin C increased plasma Vitamin E levels. ${ }^{11}$ Therefore reduction in the levels of vitamin $\mathrm{C}$ causes increased peroxidation in chronic periodontitis. The present study showed a statistically significant reduction in the plasma vitamin C levels in subjects with chronic periodontitis. This could be attributed to the protective role of vitamin $\mathrm{C}$ in an attempt to reduce the increasing reactive oxygen stress in chronic periodontitis patients. This observation is similar to various studies done. ${ }^{12,13}$ But several studies have failed to prove an association between prevalence or severity of the periodontitis diseases with low levels of vitamin $\mathrm{C}^{14,15}$ Only a weak association was found in the third National Health and Nutrition Examination Survey between low intake of vitamin $\mathrm{C}$ and periodontal diseases. ${ }^{16}$ Vitamin $\mathrm{C}$ deficiency does not cause periodontitis but it is essential in wound healing and tissue regeneration. Collagen regeneration is very essential for the maintenance of periodontal health. Vitamin C plays a role in immune modulation and synthesis of various intercellular substances like collagen.

A study showed association between Porphyromonas gingivalis is infection and low levels of plasma vitamin C which may lead to increase in colonization of this perio pathogen or disturb healing process. ${ }^{13}$

Neutrophils have the ability to concentrate ascorbic acid. It helps in bactericidal activity and enhances chemotaxis and also provides structural framework of polymorphonuclear cells. In mononuclear cells it helps in decreasing the oxidative damage to DNA. ${ }^{4}$

\section{Conclusion}

The plasma ascorbic acid level was significantly reduced in systemically healthy individuals with chronic periodontitis when compared to systemically healthy without periodontitis which could be attributed to the protective nature of this vitamin in chronic disease like periodontitis.

functions.1991;49(3):65-70.

4. Kuzmanova D, Jansen IDC, Schoenmaker T, Nazmi K, Teeuw WJ, Bizzarro S, Loos BG, Velden van der U. Vitamin C in plasma and leucocytes in relation to periodontitis. J Clin Periodontol 2012; 39 905-912.

5. Buzino R, Brodarec $A$, Jusic M ,et al. Epidemiology of angular stomatitis 
and bleeding gums. Int J Vitam Nutr Res 1973; 43:401-15.

6. Russell AL. International nutrition surveys: a summary of preliminary dental findings. J Dent Res 1963;42(1):233-244.

7. Suomalainen $K$, Sorsa $T$, Lindy $O$, Saari $H$, Konttinen $Y$, Uitto VJ. Hypochlorous acid indused activation of human neutrophil and gingival crevicular fluid collagenase can be inhibited by ascorbate. Scand J Dent Res 1991;99:397-405.

8. Clark DE, Navia JM, Manson-Hing LR, Duncan HE. Evaluation of of alveolar bone in relation to nutritional status during pregnancy. J Dent Res 1990;69:890-95.

9. Chappel ILC, Brock GR, Milward MR, Ling N, Matthews JB. Compromised GCF total antioxidant capacity in Periodontitis : cause or effect? J clin. periodontal 2007; 34: 103-10.

10. Vishakha G, Ranjan M, Kapoor A, Sachdeva S. Melatonin: Linking Periodontal Disease and Systemic Health. British Journal of Cosmetic Dentistry 2012;1(1):1-20

11. Chen, K.H. and Chang, M .L. Effect of dietary vitamin E and vitamin C on respiration and swelling of guinea pig mitochondria. J. Nutr. 108 (1978) :1616-20.

12. Panjamurthy K, Manoharan S, Ramachandran CR. Lipid peroxidation and antioxidant status in patients with periodontitis. Cellular and molecular biology letters 2005; 10:255-64.

13. Pussinen PJ, Laatikainen T, G Alfthan, Asikainen S, Jousilahti P.Periodontitis is associated with a low concentration of vitamin $\mathrm{C}$ in plasma. Clinical and diagnostic labouratory immunology sept 2003;10(5):897-02

14. Ismail Al, Burt BA, Eklund SA. Relationship between ascorbic acid intake and periodontal disease in the United States. J. Am.Dent. Assoc. 1983; 107:927-31.

15. Russel Al.International nutrition surveys: a preliminary summary of dental findings. J.Dent. Res.1963; 42:223-25.

16. Nishida M, Grossi SG, Dunford RG, Ho A W, Trevisan M, Genco R]. Dietary Vitamin C and risk of periodontal disease. J Periodontol 2000; 71:1215-23. 